\title{
Intraoral digital scanner: selection and integration in clinics
}

\author{
G. Lecocq \\ DDS, MSc Orthodontics, Private Practice
}

\section{ABSTRACT}

Acquisition of an intraoral scanner leads to an in-office revolution. Indeed, the digital workflow causes organizational changes and deeply modifies the relationship with the patients, other practitioners, and laboratory technicians. That is the reason why practitioners need to understand intraoral scanner technology and operating processes to make a convenient choice.

\section{KEYWORDS}

$3 D$ impressions, digital orthodontics, intraoral digital scanner

The digital revolution, particularly the Internet, has been welcomed into our offices in the same way that it has been welcomed into our daily life. It is accompanied by dematerialization, an unavoidable complementary process that is going to change our paradigm in terms of our organization, habits, methodologies, and professional exchanges. Consequently, in the field of orthodontics, the dematerialization of impressions, facilitated by intraoral scanners, is going to redefine our relationship with the lab and facilitate improved exchanges with colleagues and patients. This connected world forces us to modify our practice and to adapt to new protocols.

\section{INTRAORAL SCANNER TECHNOLOGY: WHAT IS THE DIGITIZATION OF DENTAL ARCHES?}

\section{Technological principles}

The fundamental principle is the transmission via the scanner of incident radiant light. This light is reflected by the surface to be scanned and then recorded by the scanner's CCD or CMOS sensor, which converts the light signal to an electrical signal. The software within the scanner will encode the information in 3D coordinates for each point. Each acquisition generates a very large number of points, the density of which defines the resolution of the 3D image $^{2,3}$. However, the internal software eliminates redundant points to standardize their density. It is therefore useless to scan an area for long durations in hopes of improving the precision. 
Orthodontic intra-oral scanners use the following four principles:

- Triangulation: the incident ray is reflected differently according to the distance with the surfaces it reflects off. It, therefore, hits a different zone on the sensor, which creates 3D coordinates (Fig. 1). This process is used by the Sirona's Cerec Bluecam system. ${ }^{1}$

- Accordion Fringe Interferometry, AFl: parallel bands of three different width of light are successively projected onto the surface to be scanned. Their distortions are recorded by an HD video camera and the 3D coordinates of the points are generated (Fig. 2). The Lythos scanner from Ormco uses this technology. ${ }^{5}$

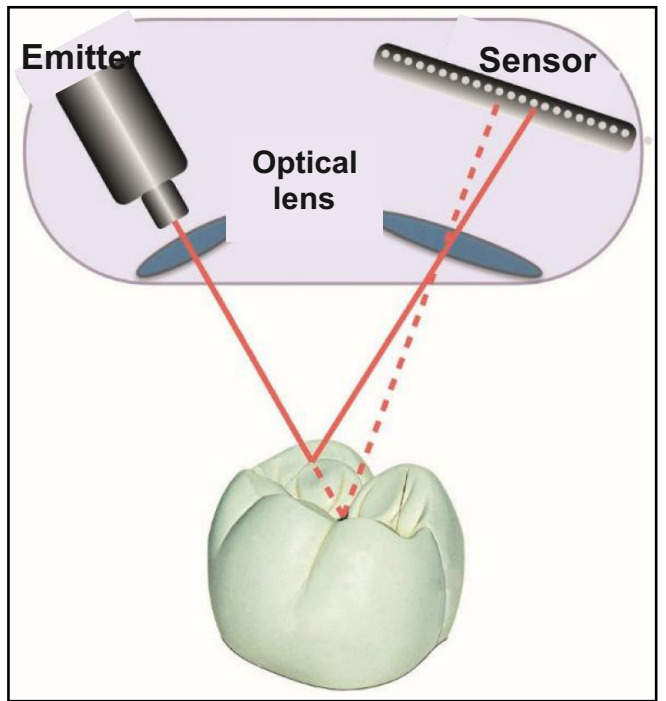

Figure 1

Triangulation laser.

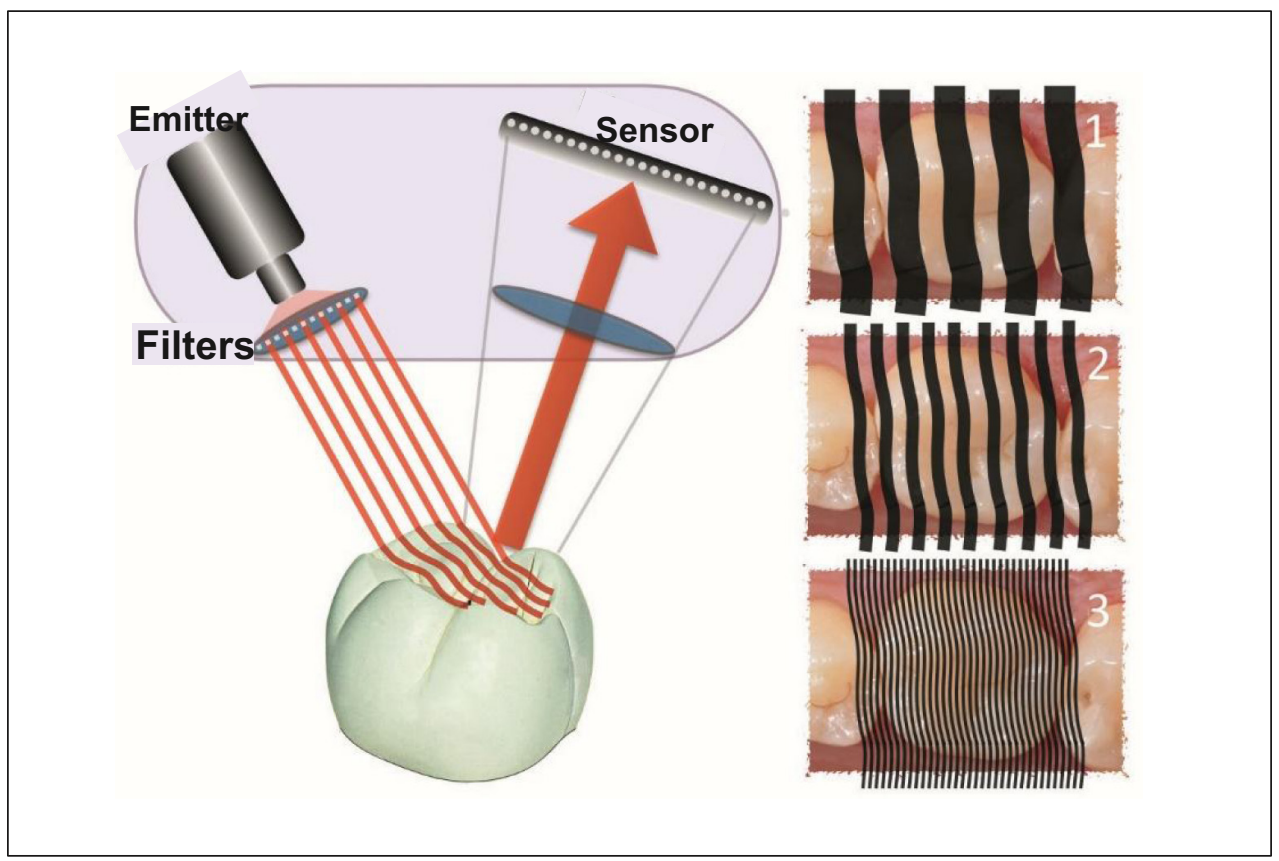

Figure 2

Accordion Fringe Interferometry - AFI 
- Parallel confocal imaging: incident light is projected through a small opening called a pinhole. Then the reflected light is returned by a mirror toward the sensor that is also equipped with a pinhole. Thus, only the points situated at the same focal distance are recorded by the sensor. The object is therefore divided into million slices, which will be computationally assembled (Fig. 3). The Cadent iTero and 3Shape Trios systems use this system. ${ }^{4}$

- Video 3D in-motion: the object is lit by a white light source or laser, and two HD video cameras that are kept at a specified distance from each other record the image from

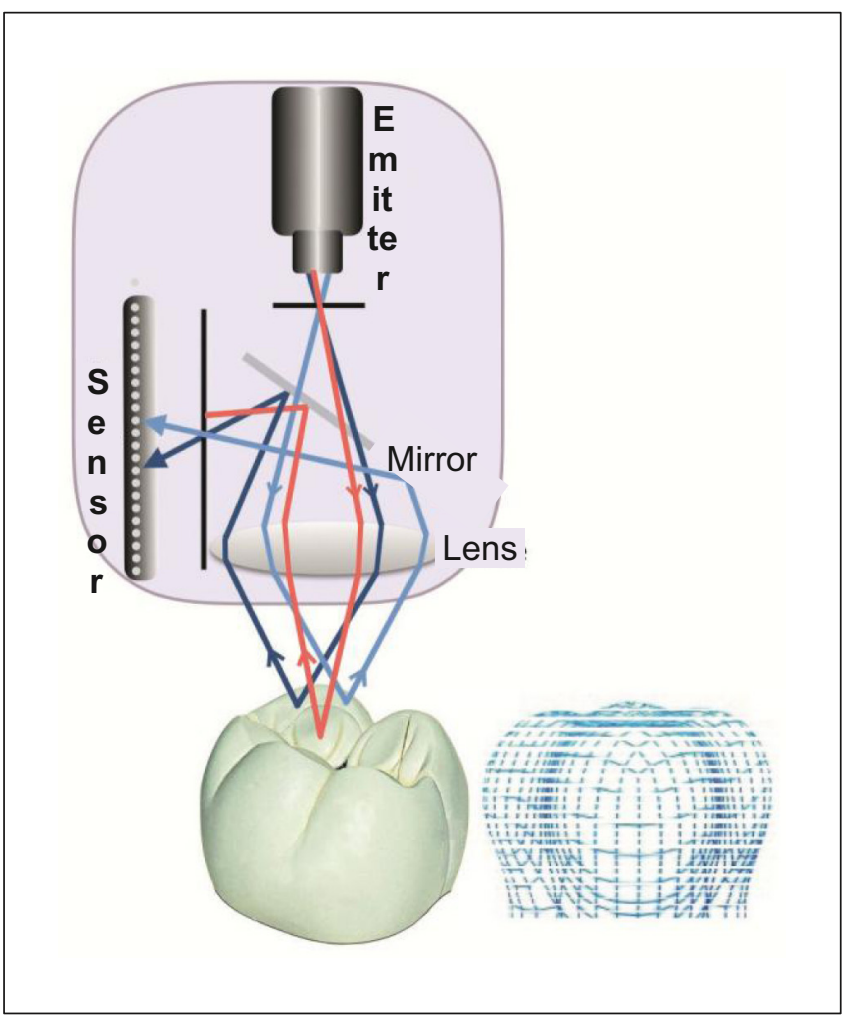

Figure 3

Parallel Confocal Imaging two perspectives. The two perspectives will be reassembled computationally to create one 3D image, as the brain does with binocular vision (Fig. 4). The True Definition system from $3 \mathrm{M}$ as well as Condor use this procedure ${ }^{4}$.

Triangulation and Accordion Fringe Interferometry have the advantage of being technologies that have long since been used in the lab to scan plaster models.

Parallel confocal imaging has the advantage of being able to measure up to $85 \%$ of all distinct angles and grooves because the incidence and reflected light beams are collinear and adopt the same linear trajectory.

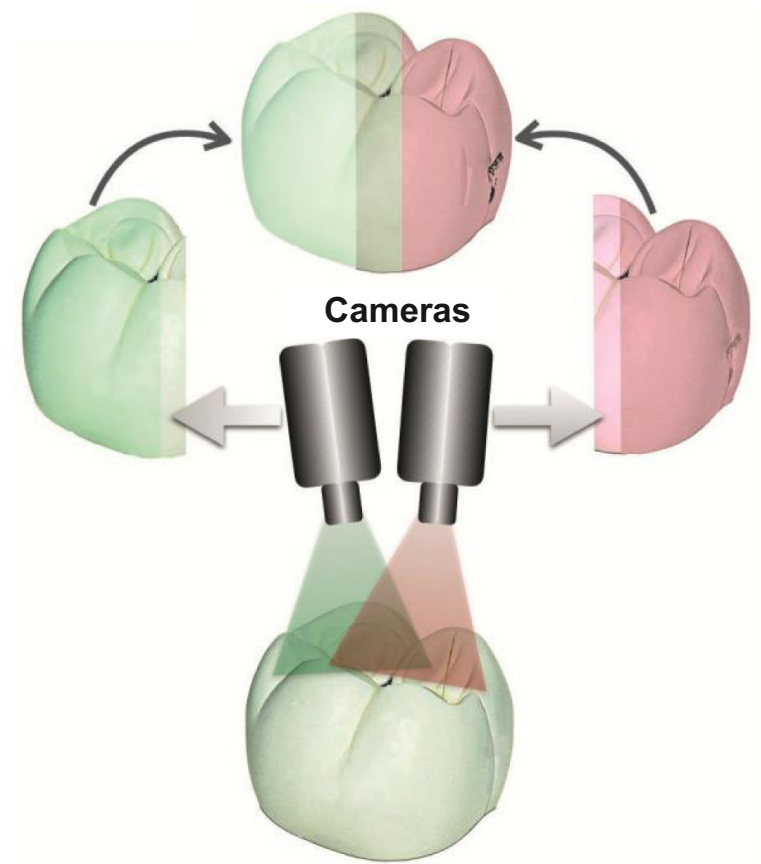

Figure 4 $3 D$ video in motion. 
In any case, the reflectivity of the scanned surface (be it enamel, ceramic, metal, or gum) must be sufficient to permit the reflection of incident light. If the reflected light is too weak no image will be recorded. It is, therefore, crucial to test the scanners on different textures and substrates or to standardize the reflectivity by dusting with a specific powder the surfaces to be recorded, which is required when using 3M's True Definition.

\section{Impression-taking technique}

It is advised that you sweep the entire dental surface following a precise path:
- On the mandible: occlusal surfaces, then lingual surfaces, and finally labial surfaces of the teeth.

- On the maxilla: occlusal surfaces, then labial surfaces, and finally palatal surfaces of the teeth, and eventually the palate if necessary.

After a disruption in acquisition, the scanner automatically detects the recapture zone if the surface contains sufficient notable points. Therefore, we usually begin with the occlusal surfaces, which are rich in topographical features. Impression-taking ends when the occlusion is recorded and this is done preferably bilaterally to increase the precision of the reconstructed occlusion.

\section{DEMATERIALIZATION AND DIGITAL FLOW: WHY USE DIGITAL IMPRESSIONS?}

The ergonomics will be modified because digital impressions are going to create a digital flow of information that is transmitted from the dental office to partner labs in a manner that is totally dematerialized, interactive and fast until the final product is received (Fig. 5).

\section{Study and pre-surgical models}

They become virtual objects that you can easily save on a hard drive and transmit via a simple click to a colleague, to health care system or to the patient, visualization being achieved via a 3D image viewer.

The virtual models can be measured, analyzed, and virtually adjusted without ever losing information because of the specialized software that renders the task less tedious.
They can either be printed in the lab or office on 3D printers to perform indirect bonding on the initial malocclusion or to obtain pre-surgical models.

\section{Virtual setup}

Digitalization of the dental arches opens the way for digital setup, which follows the same rules as manual setup. On the other hand, it has the advantage of being quickly and easily sent by email for control by the practitioners as many times as necessary. Each modification and setups are saved to the computer unlike manual setups, where each modification deletes the previous one.

Thus, the digital setup allows for the following:

- To easily test different treatment options by comparing each setup 


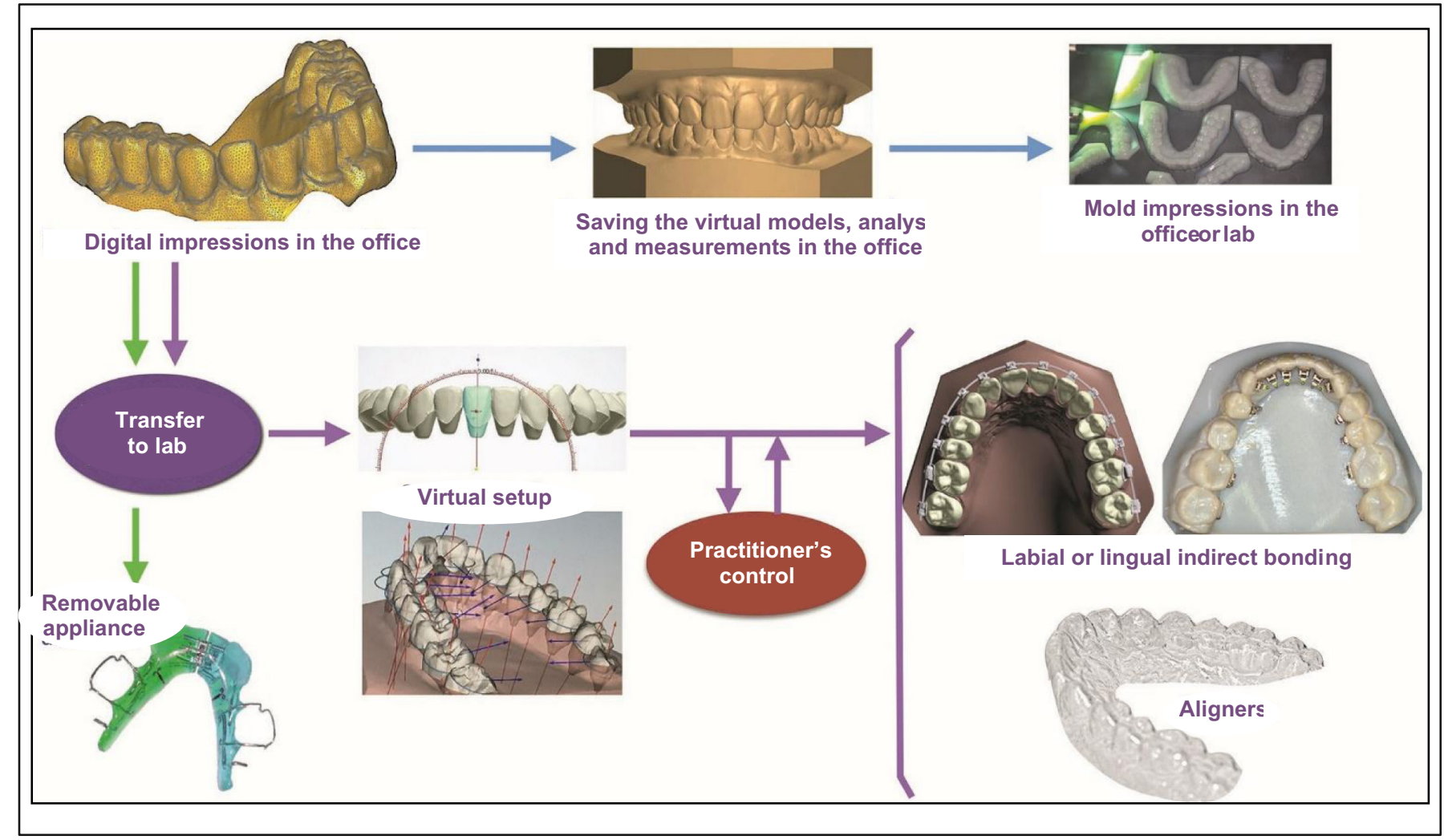

Figure 5

The digital workflow.

- To create lingual appliances via CAM

- To achieve sequencing in the treatment from the malocclusion to the final setup: each stage of treatment can be printed then thermoformed to create aligners.

\section{Removable appliance and welded devices}

The specialized lab will print the model in resin or plastic and create the removable appliance either directly on the printed model or on a duplicate in plaster. Indeed, orthodontic resin can adhere strongly to certain impression materials that are difficult to isolate.

When the welded devices are being created, the impression materials are not always adequate because they do not support the heat well. Similarly, if molar bands are necessary, information concerning the size must be confirmed in the clinic and transmitted to the lab. The lab will use equivalent bands that will be adjusted on the printed model to create the device. 


\section{INTRAORAL SCANNER INTEGRATION IN THE OFFICE: HOW AND AT WHAT COST?}

\section{Machine ergonomics}

The machine can be presented as a cart with wheels or in a compact form like the Pod Trios from 3Shape or the Lythos from Ormco, which are easier to transport from one room to another.

A touchscreen allows the operator to initiate scanning and facilitates image acquisition control; it also allows cleaning up the image and removing the undesirable scanned surfaces (lips, fingers, tongue...)

The hand piece must have a good grip and be light to carry so that it is comfortable to use. It mustn't be too tall so that it can reach the posterior areas. On the other hand, its dimension must be sufficient to allow the acquisition of the biggest possible surface for each image.

\section{Development and maintenance of the machines}

Like any new technology, the versions and machines evolve quickly. However, for the intraoral scanners it is not so much the technological principle of acquisition that is going to make the machine evolve than the softwarethe scanner's internal software-that will make improve the 3D reconstruction. Indeed, it is responsible for the elimination of redundant points, and the conservation of pertinent points that will define the resolution of the 3D image. This software also performs gap infill. Its improvement via updates will greatly contribute to improving the quality of the acquired image and 3D reconstruction.

\section{Office organization}

Digital impressions must not disorganize the office agenda. The duration of scheduled appointments must remain identical to those for traditional impressions. The duration of the appointment must consider the different steps of an appointment i.e., welcome of the patient and installation on the dental chair, time for intraoral scanning which is longer than the time displayed on the screen that doesn't count the pauses during manipulation. Then the real clinical time is therefore very different from the results displayed on the screen and commercially advertised.

\section{Saving and storing}

Storing is a crucial point. If a commercial firm is interested in selling intraoral scanners, its focus is not only limited to the act of selling the scanner but can be extended to controlling the digital flow generated by the machine. Indeed, this flow is at the center of the production and sale of devices. In addition, it seems pivotal to be able to own the files and to dispose of them as one wishes to be independent of the scanner suppliers and the by-products that they have.

That is why it is essential that the files are encoded in an open format like .stl so that they can be read by all 3D software and not strictly by the software developed by the supplier. 
Moreover, file storage can be done in a dedicated Internet space similar to the cloud made available by the supplier of the scanner. Considering this,

\section{CONCLUSION}

The digital world is insinuating itself into our offices. This way digital impressions are a revolution because the files created contain information that can be quickly and easily manipulated, facilitating the acceleration of the diagnostic process, appliance creation, etc. This works to the benefit of the ones (doctor or supplier) who will know how to operate and manage the should one have to change either the scanner or the supplier, will the files stored on their cloud still be accessible? files. In addition, the choice of intraoral scanner must be made according to how the practitioners intend to use the information and organize the workflow that they wish to establish in the office, without ignoring the scanner's mode of functioning.

Conflict of interest: The authors declare that they do not have any conflict of interest.

\section{BIBLIOGRAPHY}

1. Kravitz ND, Groth C, Jones PE, Graham JW. Intraoral Digital Scanners. J Clin Orthod 2014;6: 337-347.

2. Rolland N. Empreinte optique intra-orale sur implant pour la réalisation d'un pilier implantaire personnalisé. Thèse Doct Chir Dentaire, Univ. Toulouse III, 2014.

3. http://www.cnifpd.fr. Le guide de la CFAO dentaire

4. http://www.condorscan.com/faq

5. http://www.ormco.com/products/lythos/features.php 